Article

\title{
Helping the Visually Impaired See via Image Multi-labeling Based on SqueezeNet CNN
}

\author{
Haikel Alhichri *®D, Yakoub Bazi ${ }^{\circledR}$, Naif Alajlan and Bilel Bin Jdira \\ Advanced Lab for Intelligent Systems research (ALISR), Computer Engineering, College of Comp. and Info. \\ Sciences, King Saud University, Riyadh 11543, Saudi Arabia; ybazi@ksu.edu.sa (Y.B.); najlan@ksu.edu.sa (N.A.); \\ bjdira@ksu.edu.sa (B.B.J.) \\ * Correspondence: hhichri@ksu.edu.sa; Tel.: +966-11-469-6294
}

Received: 28 September 2019; Accepted: 30 October 2019; Published: 1 November 2019

check for updates

\begin{abstract}
This work presents a deep learning method for scene description. (1) Background: This method is part of a larger system, called BlindSys, that assists the visually impaired in an indoor environment. The method detects the presence of certain objects, regardless of their position in the scene. This problem is also known as image multi-labeling. (2) Methods: Our proposed deep learning solution is based on a light-weight pre-trained CNN called SqueezeNet. We improved the SqueezeNet architecture by resetting the last convolutional layer to free weights, replacing its activation function from a rectified linear unit (ReLU) to a LeakyReLU, and adding a BatchNormalization layer thereafter. We also replaced the activation functions at the output layer from softmax to linear functions. These adjustments make up the main contributions in this work. (3) Results: The proposed solution is tested on four image multi-labeling datasets representing different indoor environments. It has achieved results better than state-of-the-art solutions both in terms of accuracy and processing time. (4) Conclusions: The proposed deep CNN is an effective solution for predicting the presence of objects in a scene and can be successfully used as a module within BlindSys.
\end{abstract}

Keywords: visually impaired; multiple object detection; image multi-labeling; convolutional neural networks (CNN), SqueezeNet

\section{Introduction}

Visual impairment is a major problem affecting many people in the world. In 2018, the World Health Organization has estimated the number of people suffering from this impairment to be 1.3 billion, of whom 36 million are blind [1]. Visually impaired (VI) individuals face many hurdles in their daily lives. They have trouble performing many of the daily actions we take for granted such as reading text, navigating the environment, or recognizing people and objects around them. In the past, VI individuals made use of white canes or guide dogs to aid them in such tasks as navigation. They rely on braille systems for reading and learning. They also rely on other people to help them from time to time especially with more complex tasks. However, technology can offer many solutions for VI individuals with a more consistent and contentious service. Early examples include refreshable braille displays, which allowed the VI to type and view text [2,3]. It is a kind of keyboard composed of cells that can be refreshed with braille characters to reflect the text on a screen. Another solution is the screen reader, which is a type of text-to-speech supplication, developed by researchers at IBM $[4,5]$. Other types of technological solutions include the use of robotics to aid the VI in navigation and to serve as a home assistant $[6,7]$.

Nowadays, technology solutions based on advances in computer vision and machine learning has evolved into one of the most exciting areas of technological innovation today. The overwhelming majority of the contributions can be categorized under one of the two categories confined to navigation 
(i.e., by allowing more freedom in terms of mobility, orientation, and obstacle avoidance) and recognition (i.e., by providing the blind person with information related to the nature of objects encountered in his/her context). The category of research work focusing on the recognition task for blind people consists of several notable works [8-14]. However, many challenging issues are still unresolved due to the complex nature of indoor scenes and the need to recognize many types of objects in a short period of time for the system to be useful to a blind person. One way to reduce the complexity of the problem is not to care about providing the exact location of objects in the scene to the blind person. Instead, only detect the mere presence of the objects in the scene. Then, this problem becomes similar to what is known as image multi-labeling, or in other words assigning an image to many classes instead of just one. In this context, Mekhalfi et al. proposed a method for the detection of the presence of a set of objects based on the comparison to a set of stored images with known object contents [8]. To compare the query image to the stored images, the authors proposed to use features based on scale invariant feature transform (SIFT), the notion of a bag of words (BOW), and principal component analysis (PCA). Besides, using hand-crafted features which may not be able to describe the images well, their method relies on selecting a set of representative images to be used for comparison. However, in addition, comparing to this set of images for every query image is computationally expensive. The authors in [9] proposed another method which exploits Compressive Sensing (CS) theory for image representation and a semantic similarity metric for image likelihood estimation through learned Gaussian Process Regression (GPR) models. Malek et al. [12] used three low-level feature extraction algorithms, namely the histogram of oriented gradient (HOG), the BOW, and the local binary pattern (LBP). They merged these features using a deep learning approach, in particular an auto encoder neural network (AE), to create a new high-level feature representation. Finally, a single layer neural network was used to map the AE features into image multi-labels.

Recently there has been a major development in the field of computer vision for object detection and recognition-based deep learning approaches, in particular the set of convolutional neural networks (CNNs) [15-18]. Deep learning is a family of machine learning based on learning data representation. The learning can be supervised or unsupervised depending on the adopted architecture. The most famous deep architectures are the stacked AutoEncoder (SAE) [19], which consists in a concatenation of many AutoEncoders (AEs), a deep belief network (DBN) [20], which is based on a series of restricted Boltzmann machines (RBMs), and CNNs [21]. Deep CNNs have achieved impressive results on a variety of applications including image classification [22-25], object detection [15-18], and image segmentation [26]. Thanks to their sophisticated structure, they have the ability to learn powerful generic image representations in a hierarchical way compared to state-of-the-art shallow methods based on handcrafted features. Modern $\mathrm{CNNs}$ are made up of several alternating convolution and pooling layers followed by some fully connected layers. The feature maps produced by the convolution layers are usually fed to a nonlinear gating function such as the sigmoid function or the more advanced rectified linear unit (ReLU) function. The output of this activation function can then be further subjected to normalization (i.e., local response normalization) to help with generalization. The whole CNN architecture is trained end-to-end using the backpropagation algorithm [27].

Like any other classification model that learns a mapping between input data and its labels, CNNs are prone to overfitting. Overfitting refers to a model that learns the training data too well, but when it comes to the unseen testing data, it fails. Overfitting is more likely with nonparametric and nonlinear models that have more flexibility when learning a target function. CNNs are nonlinear models that have large amounts of free weights that can be learned. Thus, they have more flexibility to learn training data too well. They can learn the detail and noise in the training data to the extent that it negatively impacts the performance of the model on unseen testing data. When we have large amounts of labeled training data, then overfitting is drastically reduced. However, with small training data, overfitting is a major problem for deep machine learning. In this case, it has been shown that it is more interesting to transfer knowledge from CNNs (such as AlexNet [28], VGG-VD [29], GoogLeNet [30], and ResNet [24]) pre-trained on an auxiliary recognition task with a very large amount of labeled 
data instead of training a CNN from scratch [31-34]. The possible transfer learning solutions include fine-tuning the pre-trained $\mathrm{CNN}$ on the labeled data of the target dataset or to exploit the CNN feature representations with an external classifier. We refer the reader to [32] where the authors introduce and investigate several factors affecting the transferability of these representations.

In this work, we build a real-time deep learning solution for the image multi-labeling problem. The image multi-labeling solution is a module within a larger system, called BlindSys, that helps the visually impaired see. Through the image multi-labeling module, the visually impaired can become aware of the presence of a given set of objects in his environment. Our proposed deep learning solution is based on a light-weight pre-trained CNN called SqueezeNet that has less than 1.5 million weights. We improve the SqueezeNet architecture by resetting the last convolutional layer to free weights, replacing its activation function from a ReLU to a LeakyReLU, and adding a BatchNormalization layer thereafter. We also replace the activation functions at the output layer from softmax to linear functions. These adjustments help with the generalization capability of the network as demonstrated by the experimental results. Due to its small size, the proposed deep CNN for image multi-labeling can be trained using a fine-tuning approach with reasonable computational resources. Therefore, the main contributions of this work can be summarized as follows:

1) A novel deep learning solution for image multi-labeling based on knowledge transfer from the pre-trained SqueezeNet CNN.

2) The proposed $\mathrm{CNN}$ architecture improves the SqueezeNet $\mathrm{CNN}$ by using the more advanced LeakyReLU activation function and the BatchNormalization technique. It also reduces the number of weights (to less than 900,000) to combat overfitting.

3) The proposed deep solution uses a fine-tuning approach to adapt the $\mathrm{CNN}$ to the new target dataset.

The rest of the paper is organized as follows. In Section 2, we give a full description of the methodology, including the problem formulation, the CNN theory, and the proposed deep CNN solution based on SqueezeNet. Section 3 presents the datasets and the experimental results. Finally, concluding remarks are given in Section 4.

\section{Materials and Methods}

First, this section defines the problem at hand formally. We then explain how to solve image multi-labeling using pre-trained convolutional neural networks in Section 2.2. Finally, we present our proposed solution based on the pre-trained model known as SqueezeNet.

\subsection{Problem Formulation}

Let $\left\{\mathbf{I}_{i}, \boldsymbol{y}_{i}\right\}_{i=1}^{n}$ be a training set where $\mathbf{I}_{i} \in \mathcal{R}^{w \times h \times 3}$ is an image of size $w \times h \times 3$, and $y_{i} \in \mathcal{R}^{1 \times c}$ is its corresponding "object indicator" vector of size $c$, which is equal to number of objects of concern. Vector $y_{i}$ is composed of a binary sequence where one indicated the presence of an object in the scene, while zero indicated otherwise. Let also $f_{l}^{\mathrm{CNN}}, l=1, \ldots, L$ be a CNN model with $L$ layers pre-trained on a large amount of labeled auxiliary images from a different domain. Here, $f_{l}^{\mathrm{CNN}}$ refers the output of the $l^{\text {th }}$ layer. Our aim is to develop a deep learning system that can map the test images $\left\{\mathbf{I}_{i}\right\}_{i=1}^{m}$ to binary vectors $y_{i}$ (which indicates the presence or no presence of objects) based on the available training set as well the pre-trained $\mathrm{CNN}$ model. Figure 1 clarifies the image multi-labeling problem further. 


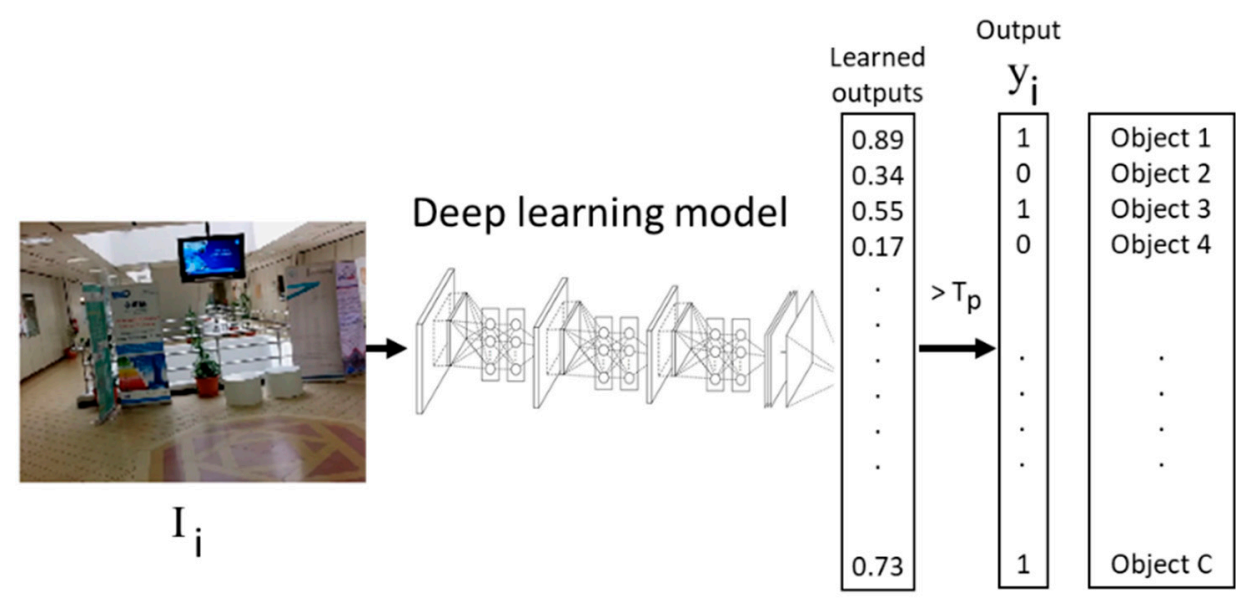

Figure 1. Overview of the image multi-labeling problem.

\subsection{Deep CNN Architectures}

Deep learning CNNs are composed of several layers of processing, each comprising linear as well as non-linear operators, which are learnt jointly, in an end-to-end way, to solve specific tasks $[10,11]$. Specifically, deep CNNs are commonly made up of four types: 1) a convolutional layer; 2) a normalization layer, 3) a pooling layer, and 4) a fully connected layer. The convolutional layer is the core building block of the $\mathrm{CNN}$, and its parameters consist of a set of learnable filters. Every filter is small spatially (along width and height), but extends through the full depth of the input image. The feature maps produced via convolving these filters across the input image are fed to a non-linear gating function such as the sigmoid or ReLU functions [28].

Regarding the pooling layer, it takes small rectangular blocks from the convolutional layer and subsamples it to produce a single output from each block. There are several ways to perform pooling, such as taking the average or the maximum, or a learned linear combination of the values in the block. The main two reasons of using this layer are 1) to control overfitting and 2) to reduce the amount of parameters and computation in the network. After several convolutional and pooling layers, the high-level reasoning in the neural network is done via fully connected layers. A fully connected layer takes all neurons in the previous layer and connects it to every single neuron it has. All the weights in the CNN are learned using the well-known back-propagation technique.

\subsection{Image Multi-Labeling Based on a Pre-Trained CNN}

Typically, early deep leaning solutions use a pre-trained $\mathrm{CNN}$ as an extractor of high level features. The complete learning model then uses extra network layers (usually fully connected layers) to adapt the network to another problem. Given the deep nature of a CNN, one can extract features at different layers. Thus, given an image $\mathbf{I}_{i}$, we can generate a feature representation vector $x_{i} \in \mathcal{R}^{D}$ of dimension $D$ as follows:

$$
x_{i}=\mathrm{f}_{k}^{\mathrm{CNN}}\left(\ldots \mathrm{f}_{2}^{\mathrm{CNN}}\left(\mathrm{f}_{1}^{\mathrm{CNN}}\left(\mathbf{I}_{i}\right)\right)\right), i=1, \ldots, n
$$

where $\mathrm{k}$ is a layer within the network. For example, we can use the output of the hidden fully connected (FC) layer just before the last output layer (which is used to produce a classification result in the original $\mathrm{CNN}$ framework), to represent the training and test images. In this case, $\mathrm{k}=\mathrm{L}-1$, where $\mathrm{L}$ is the total number of layers in the network. Next, the extracted feature is fed into a fully connected (dense) layer that has free trainable weights that can be trained on the new dataset at hand. Figure 2 illustrates this typical architecture. 


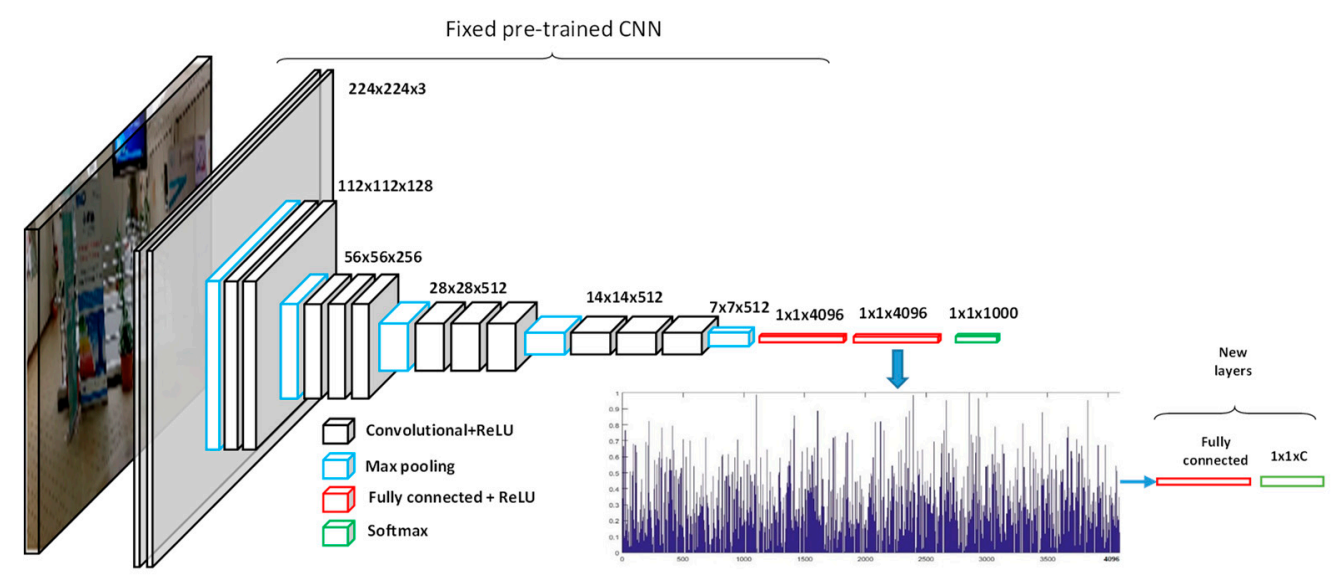

Figure 2. Feature extraction using a pre-trained Convolutional Neural Network (CNN) model, where a new fully connected layer followed by a softmax layer with $C$ neurons (equal to number of classes) are added for learning.

In the extra hidden FC layer, the input $\boldsymbol{x}_{i} \in \mathfrak{R}^{D}$ is mapped to the hidden representation $\boldsymbol{h}_{i} \in \mathfrak{R}^{S}$ of dimension $S$ through the nonlinear activation function $f$ as follows:

$$
\boldsymbol{h}_{i}=\mathrm{f}\left(\mathbf{W}^{(h)} \cdot \boldsymbol{x}_{i}+\boldsymbol{b}^{(h)}\right)
$$

where $\mathbf{W}^{(h)} \in \mathfrak{R}^{S \times D}$ is the weight matrix, and $\boldsymbol{b}^{(h)} \in \mathfrak{R}^{S}$ is the bias vector. Similarly, the output vector $y_{i}$, is computed through a nonlinear activation function $\mathrm{F}$ as follows:

$$
\boldsymbol{y}_{i}=\mathrm{f}\left(\mathbf{W}^{(o)} \cdot \boldsymbol{h}_{i}+\boldsymbol{b}^{(o)}\right)
$$

Typical choice of the activation function is the sigmoid function, i.e., $\mathrm{f}(v)=1 /(1+\exp (-v))$, or the more advanced functions called ReLU or LeakyReLU shown in Figure 3.
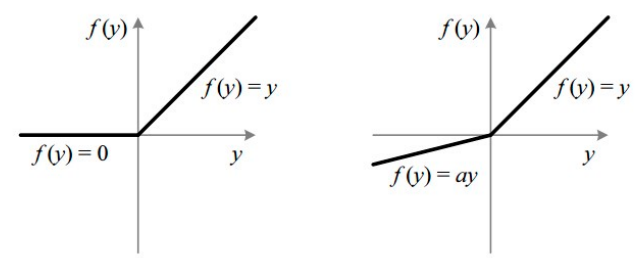

Figure 3. State-of-the-art activation functions.

(a) ReLU activation function; (b) LeakyReLU activation functions.

In order to utilize this pre-trained CNN to solve a new classification problem, we need to build the output layer so that it has as many neurons as the number of classes (typically the original CNN has an output layer with size 1000). Furthermore, for a typical classification problem, the output layer uses a softmax activation function and the categorical cross entropy loss function. Given the outputs $\boldsymbol{h}_{i}^{(1)}$ of the last layer before the output layer, the softmax activation function produces an estimate of the posterior probability for each class label $c=1,2, \ldots, C$ as in Equation (4):

$$
p\left(\hat{y}_{i}=c \mid \boldsymbol{x}_{i}\right)=\frac{\exp \left(\left(\boldsymbol{w}_{c}^{(2)}\right)^{\mathrm{T}} \boldsymbol{h}_{i}^{(1)}\right)}{\sum_{j=1}^{\mathrm{C}} \exp \left(\left(\boldsymbol{w}_{j}^{(2)}\right)^{\mathrm{T}} \boldsymbol{h}_{i}^{(1)}\right)}
$$


where $\boldsymbol{W}^{(2)}=\left[\boldsymbol{w}_{1}^{(2)} \boldsymbol{w}_{2}^{(2)} \ldots \boldsymbol{w}_{C}^{(2)}\right] \in \mathfrak{R}^{d \times C}$ are the weights of the softmax layer and the superscript $(\cdot)^{\mathrm{T}}$ refers to the transpose operation. This softmax activation function turns the outputs of the neurons in the last layer into probabilities by ensuring that their total sum is equal to one. This is important for classification problems because we expect only one neuron to output one (because each input image must be assigned to one class) while the rest will be zero. However, it is not appropriate for image multi-labeling because we expect many neurons to output the value one and hence the sum will not be equal to one. Thus, we use other activation functions, such as linear or sigmoid, in the output layer and optimize the mean squared error (MSE) loss function, similar to deep models for regression problems. The linear activation is more appropriate because it has a derivative equal to one, which is preferable for the backpropagation algorithm used during training. Furthermore, the linear function is not limited to the interval $[0,1]$ like the sigmoid function, so each output neuron is free to estimate values below or above the correct outputs of zero or one.

To train the neural network, we use the back propagation technique and minimize the following cost function [35]:

$$
\mathcal{L}_{2}\left(\theta_{N N}\right)=\frac{1}{2 n} \sum_{i=1}^{n} \sum_{k=1}^{C}\left\|\boldsymbol{y}_{i}-\theta_{N N}\left(\mathbf{x}_{i}\right)\right\|^{2}+\frac{\lambda}{2}\left(\sum_{l=1}^{H}\left\|\mathbf{W}^{(h)}\right\|_{F}^{2}+\sum_{o=1}^{c}\left\|\mathbf{W}^{(o)}\right\|_{F}^{2}\right)
$$

where $\theta_{N N}\left(\mathbf{x}_{i}\right)$ is the output of the NN for an input $\mathbf{x}_{i}, \mathbf{W}^{(h)}$ and $\mathbf{W}^{(o)}$ are the weights of the hidden layer and the output layer, respectively, and $H$ is the size of the hidden layer. The first term of (1) refers to the mean squared error between the estimated labels and the training labels, while the second term is the weight decay penalty that helps to prevent overfitting. Note also that $n$ is the number of training samples, and $C$ is the output vector size (the number of objects to be recognized). The estimation of the vector of parameters $\theta_{N N}=\left\{\mathbf{W}^{(h)}, \mathbf{W}^{(o)}\right\}$ of the NN starts by initializing the weights to small random values. The cost (1) is then minimized with a min-batch gradient descent algorithm [36].

At test time, we feed each test image to the $\mathrm{CNN}$ and assume that an output node $j$ having a predicted value larger than a given threshold (for example, 0.5) indicates the presence of the object $j$. We call this parameter the presence threshold $T_{p}$.

\subsection{The Proposed Solution Based on the SqueezeNet CNN}

During training on the new given dataset, the weights of the pre-trained CNN are either fixed or allowed to be updated. The latter case is known as fine-tuning and is obviously more time-consuming due to the large number of weights that need to be optimized. For example, successful CNNs such as AlexNet CNN [28] and the VGG-very-deep-16 CNN model [29] have more than 60 and 138 million weights, respectively. This makes them quite difficult to fine-tune for typical low-end workstations. Furthermore, because this image multi-labeling module is running on a mobile device, computational resources are limited, and hence execution time of the solution at test time must be as fast as possible. That is why, we have selected a much lighter pre-trained CNN, called SqueezeNet, that has less than 1.5 million weights, yet its accuracy is close to that of the AlexNet CNN [28].

Figure 4a shows the architecture of SqueezeNet. It has 26 convolutional layers (with 34 other types of layers) and about 860 million multiply-accumulate operations compared to 1140 million for AlexNet [28]. With the help of model compression techniques, SqueezeNet has a file size of less than $0.5 \mathrm{MB}$ (510 times smaller than AlexNet). SqueezeNet also accepts images of variable size (as low as $48 \times 48$ ) without the need for resizing, albeit $224 \times 224$ is still the ideal size. All these properties make it suitable for us to build and train our solution. 


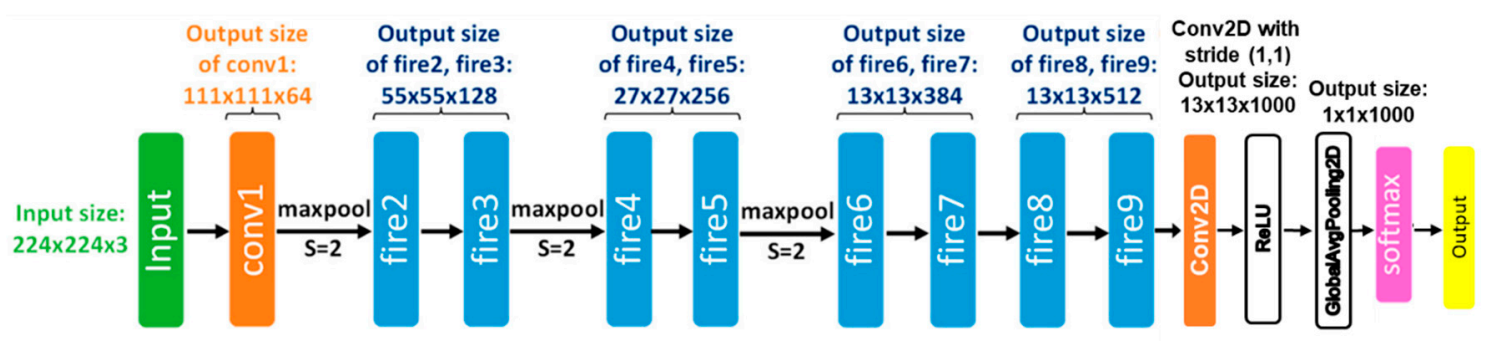

(a)

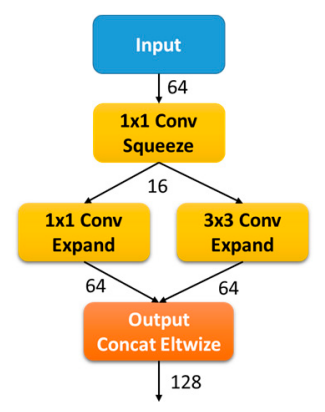

(b)

Figure 4. Overview of the SqueezeNet architecture. (a) Overall architecture; (b) the fire module with squeeze and expand layers.

The building block of SqueezeNet is called the fire module, illustrated in Figure $4 \mathrm{~b}$. It contains two layers: a squeeze layer and an expand layer. A SqueezeNet stacks a bunch of these fire modules and a few pooling layers. The squeeze layer decreases the size of the feature map, while the expand layer increases it again. As a result, the same feature map size is maintained. Another pattern is increasing depth, while reducing the feature map size to obtain a high level abstract. This is accomplished through increasing the number of filters and using a stride of two in the convolutional layers.

In this work, we propose a solution for the image multi-labeling problem based on fine-tuning an improved version of a pre-trained SqueezeNet CNN. In particular, we designed two improved SqueezeNet models to be used with the fine-tuning approach. We also build a base model or Model 1, illustrated in Figure 5a, that uses the pre-trained SqueezeNet as a feature extractor (i.e. its weights are fixed) and adds a fully connected layer for adapting to the given dataset. The other two improved models, referred to as Model 2 and Model 3, are illustrated in Figure 5b,c, respectively. In Model 2, we fine-tune Model 1, by making all weights of the SqueezeNet layers trainable. In Model 3, we get rid of the extra FC layer of Model 1 and reset the last convolutional layer of the SqueezeNet model to random weights. Removing the FC layer has the advantage of reducing the weights of the deep network, which consequently decreases computational costs. Resetting the weights of the last convolutional layer improves the generalization capability and affords a greater chance for the network to adapt to the new dataset. Furthermore, in all models above, we replaced the ReLU activation function of the hidden layer with new weights with the more advanced LeakyReLU activation function. We then inserted a BatchNormalization layer thereafter to fight overfitting and improve generalization capability of the network. This was not present in the original SqueezeNet model.

The BatchNormalization layer was introduced back in 2014 in the second version of GoogLeNet by Szegedy et al. [37]. BatchNormalization is similar to the concept of standardization (make the data have zero mean and sometimes also unit standard deviation) of the network input, which is a common thing to do before using a CNN on any data. BatchNormalization does the same thing inside the CNN. Specifically, we add a normalization layer after the convolutional layer, which makes the data in the current training batch (data is divided in batches to make sure it fits in computer RAM) follow a normal distribution. This way the model focuses on learning the patterns in the data and is not misled 
by large values in it. This in turn helps with combating overfitting and increasing the generalization ability of the network. Another advantage of the BatchNormalization technique is that it allows the model to converge faster in training and hence allows us to use higher learning rates.

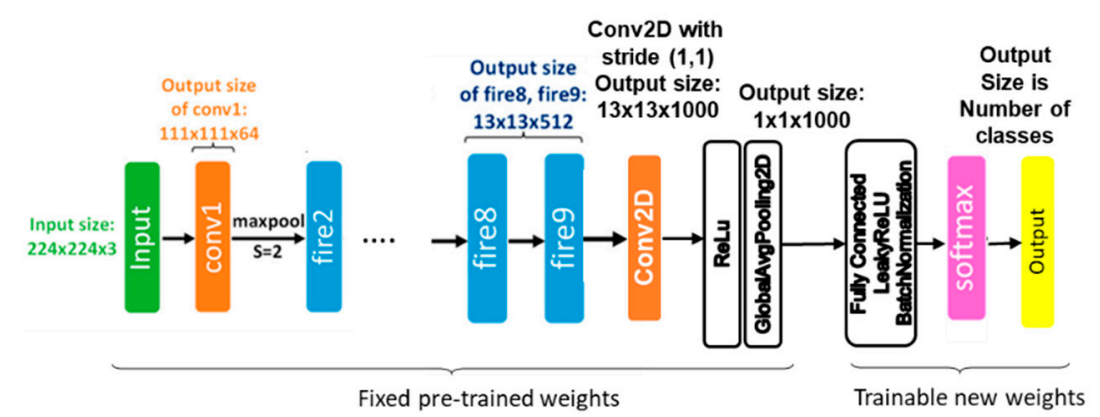

(a)

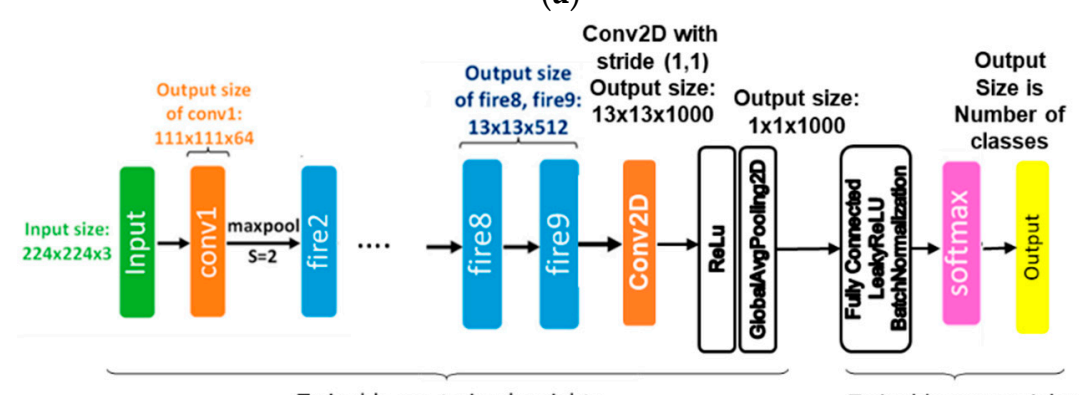

Trainable pre-trained weights

Trainable new weights

(b)

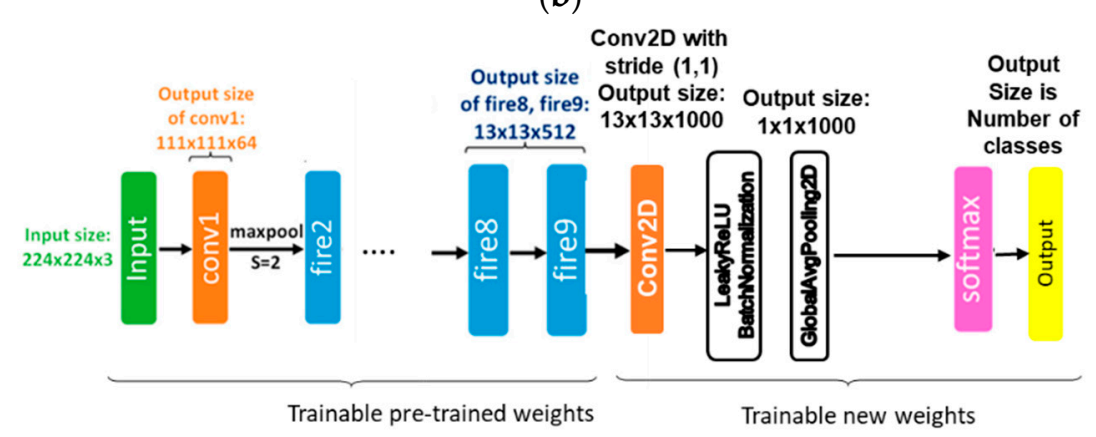

(c)

Figure 5. Proposed architecture using the SqueezeNet pre-trained model. (a) Model 1: base model with fixed pre-trained SqueezeNet. (b) Model 2: fine-tuned Model 1. (c) Model 3: fine-tuned SqueezeNet with new random weights for the last convolutional layer (after fire9).

The proposed solution fights overfitting by transferring the knowledge from a CNN pre-trained on a large auxiliary dataset (namely ImageNet), instead of training it with random initial weights. In addition, we also combat overfitting by reducing the number of free weights and hence reducing the flexibility of the network so that it does not learn the small details or noise in the dataset. Finally, the new BatchNormalization technique introduced inside the network improves the generalization ability of the network and fights overfitting, because it forces all feature values inside the network to be within the same small range (zero to one), instead of having the flexibility to use feature values as large as it wants in order to fit the training data.

\section{Experimental Results}

This section presents the experimental work of this paper. As we mentioned before, this work is part of a larger project, called BlindSys, that aims at designing an IT system to help the visually impaired 
perform important tasks in their lives. This system is also the source of one of the datasets used in this paper. As a result, we first present this IT system in the next subsection. After that, we describe the image datasets used in the paper and some experimental setup issues. Finally, we present the results and compare them to state-of-the-art methods.

\subsection{BlindSys: A Smart IT Solution to Assist the Visually Impaired}

Figure 6 shows an overview of the BlindSys system for guiding VI people and helping them recognize objects and text in indoor environments. The BlindSys hardware is composed of a high resolution camera that is mounted at the chest of the VI person, a wireless headphone for voice communication with the VI user, and a mobile device that houses the BlindSys software and enables interface with the system.

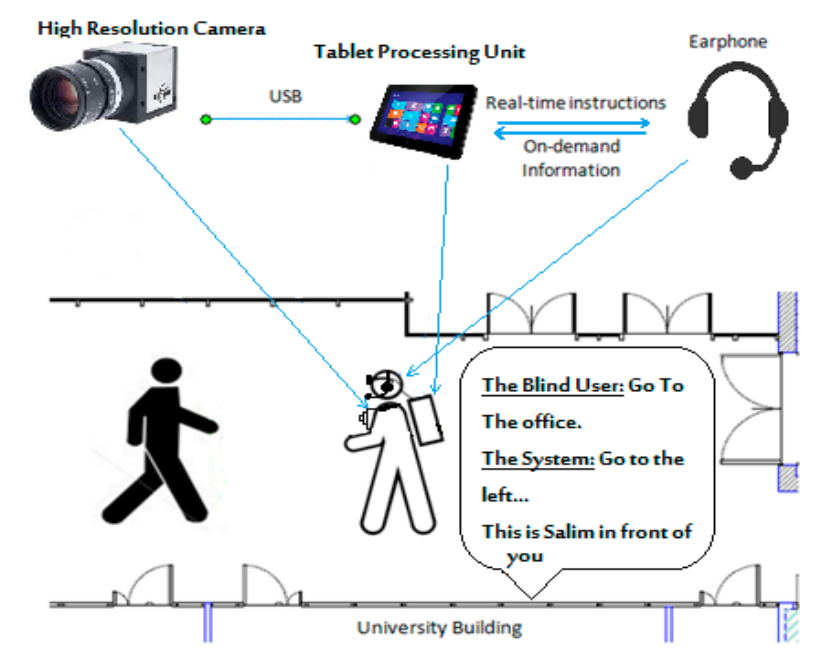

Figure 6. Overview of the BlindSys system for the visually impaired (VI). Diagram shows the camera on the chest, tablet, and headphones for voice communication.

The VI user can use the system via voice commands or through a graphic user interface (GUI) to select one of four functions: 1) indoor navigation, 2) text detection and recognition, 3) scene description, or 4) face detection and recognition. Indoor navigation operates on the video stream in real time, while the other functions operate on a single image captured upon request from the video stream. To ease operation of the system, the mobile device has the simple GUI shown in Figure $7 \mathrm{~b}$, where the whole screen is divided into four large buttons. The VI user can press the buttons simply by touching an area close to the corners of the device. In order to avoid accidental touching, the VI user should tap the button twice consecutively. BlindSys has another main GUI, shown in Figure 7a, that can be used for testing and operator support. All processing results are communicated to the VI user via voice using text-to-speech synthesis.

The BlindSys software is based on three main parts. 1) The main application contains the application GUI and the business logic under it that manipulates all the gathered information to give navigation details to the user (video extracted information, IMU (inertial measurement unit) information, and so on). 2) Auxiliary applications that are independent from the main application are responsible for performing other functions such as OCR, face recognition, scene description, and so on. 3) Libraries are used to implement the different parts of the main application or the auxiliary applications. They are linked to the application statically or dynamically. 


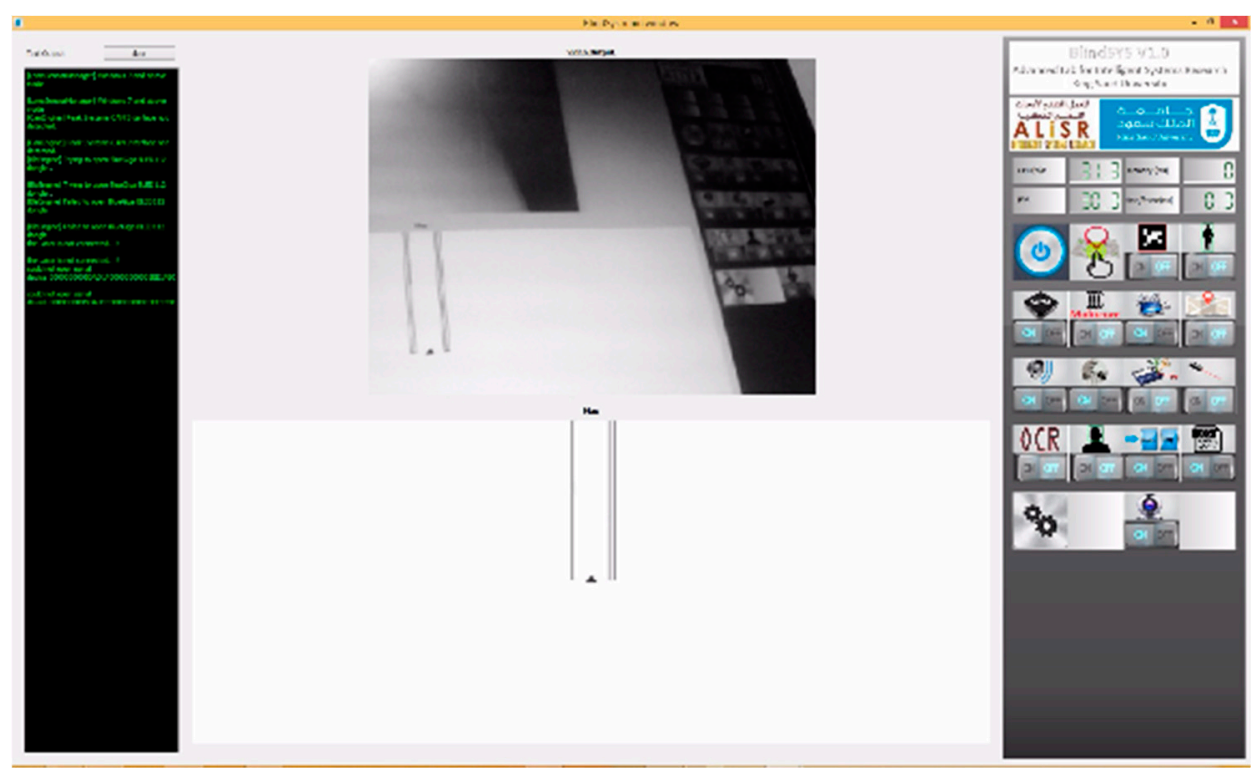

(a)

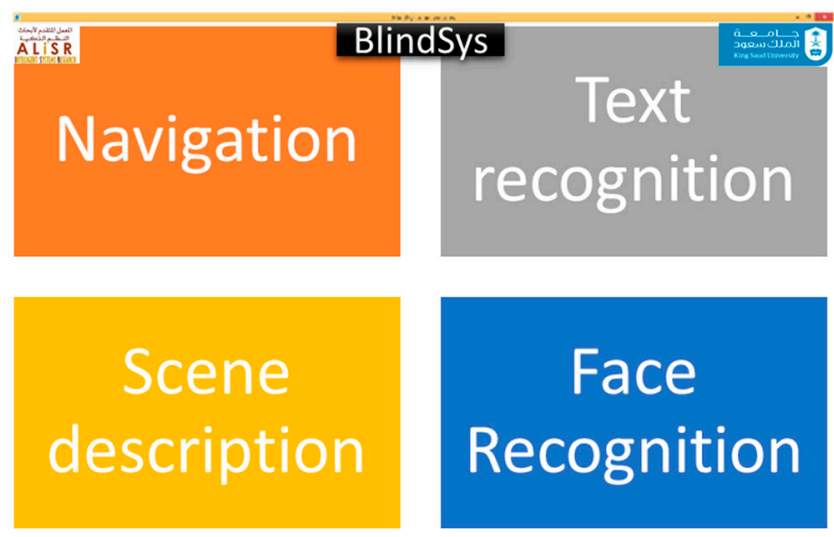

(b)

Figure 7. Graphic user interface (GUI) of the prototype implemented to collect dataset images at King Saud University. (a) Detailed GUI for testing and operator support. (b) Simple GUI on the mobile device to be used by the VI.

The scene description module can detect the presence of a set of objects in a scene image without giving exact locations. The module can detect a set of 15 objects that are commonly found in an indoor environment.

\subsection{Dataset Description}

The experiments in this paper use four datasets of multi-labeled images for evaluating the efficiency of the proposed deep learning solution. The first two datasets pertain to the college of the computer and the information sciences building at the King Saud University (KSU), Saudi Arabia. They have been collected by our team using the BlindSys system described in Section 3.1. The second two datasets were collected by the authors of [8] in two different buildings of the University of Trento, Italy. The cameras used to capture these images are from a company called IDS-imaging [38]. For the KSU datasets, the camera used was a UI-1220SE -C-HQ, USB 2.0, CMOS, $87.2 \mathrm{fps}, 752 \times 480,0.36 \mathrm{MPix}$, $1 / 3^{\prime \prime}$, ON Semiconductor, Global Shutter. For the UTrento datasets, the camera used was a UI-1240LE -C-HQ, USB 2.0, CMOS, 25.8 fps, $1280 \times 1024,1.31$ MPix, 1/1.8", e2v, Global Shutter, Global Start Shutter, Rolling Shutter. Both cameras were equipped with a KOWA LM4NCL 1/2", 3.5 mm, F1.4 manual IRIS 
C-Mount lens from RMA Electronics Inc. [39]. The details of these four datasets are given in Table 1, which also presents the list of objects considered in every dataset.

Table 1. Details of the four datasets of multi-labeled images used in the work.

\begin{tabular}{ccl}
\hline Dataset & Train/Test Sizes & \multicolumn{1}{c}{ Objects Considered } \\
\hline KSU1 & $161 / 159$ & $\begin{array}{l}\text { pillar, fire extinguisher/hose, trash can, chairs, external door, hallway, } \\
\text { self-service, reception, didactic service machine, display screen, board, } \\
\text { stairs, elevator, laboratory, internal door } \\
\text { board, fire extinguisher/hose, trash cans, chairs, external door, didactic } \\
\text { service machine, self-service, reception, cafeteria, display screen, pillar, } \\
\text { stairs, elevator, prayer room, internal door } \\
\text { external window, board, table, external door, stair door, access control } \\
\text { reader, office, pillar, display screen, people, ATM, chairs, bins, internal } \\
\text { door, and elevator } \\
\text { stairs, heater, corridor, board, laboratories, bins, office, people, pillar, } \\
\text { elevator, reception, chairs, self-service, external door, and display screen }\end{array}$ \\
UTrento1 & $56 / 88$ & \\
UTrento2 & $61 / 70$ &
\end{tabular}

The King Saud University 1 (KSU1) dataset is composed of 320 images, divided into 161 training images and 159 testing images. The King Saud University 2 (KSU2) dataset is composed of 174 images, divided into 86 training images and 88 testing images. The University of Trento 1 (UTrento1) dataset accounts for a total of 130 images acquired in two separate daytimes (morning and evening), and these were split into 58 training images (i.e., for training the $\mathrm{NN}$ model) and 72 for testing purposes. Finally, the University of Trento 2 (UTrento2) dataset is composed of 131 images, divided into 61 training images and 70 testing images. It is noteworthy that the training images for all datasets were selected in such a way to cover all the predefined objects in the considered indoor environment. Thereupon, we have selected the objects deemed to be the most important ones in the considered indoor environments. Figures 8 and 9 show sample images from the KSU2 and UTrento2 datasets with the list of objects contained within. Furthermore, Figure 10 presents the number of occurrences of objects in the training set and the test set of every dataset.
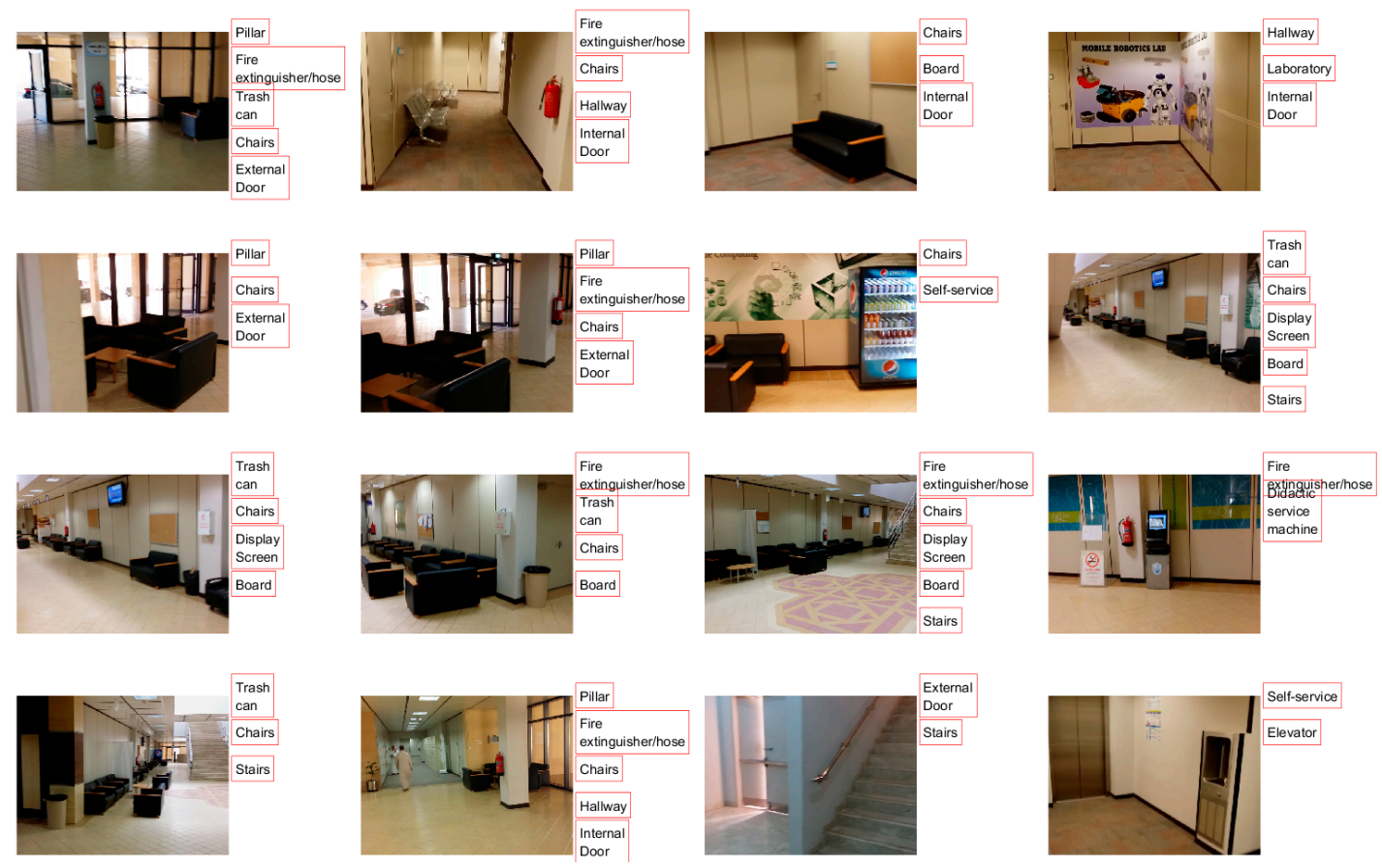

Figure 8. Sample images from the King Saud University 2 dataset (KSU2) and the corresponding labels (objects). 

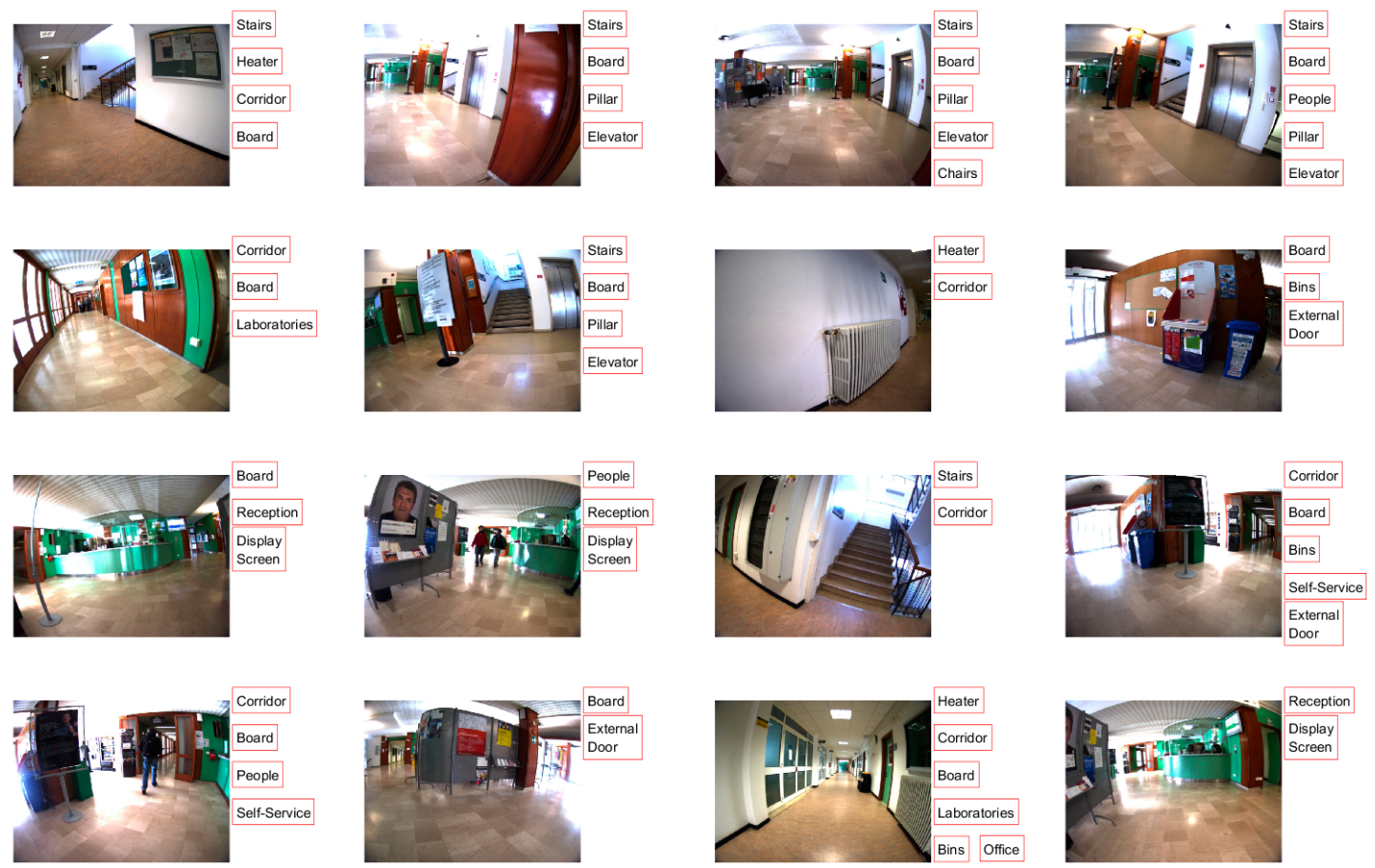

Figure 9. Sample images from the University of Trento 2 dataset (UTrento2) and the corresponding labels (objects).
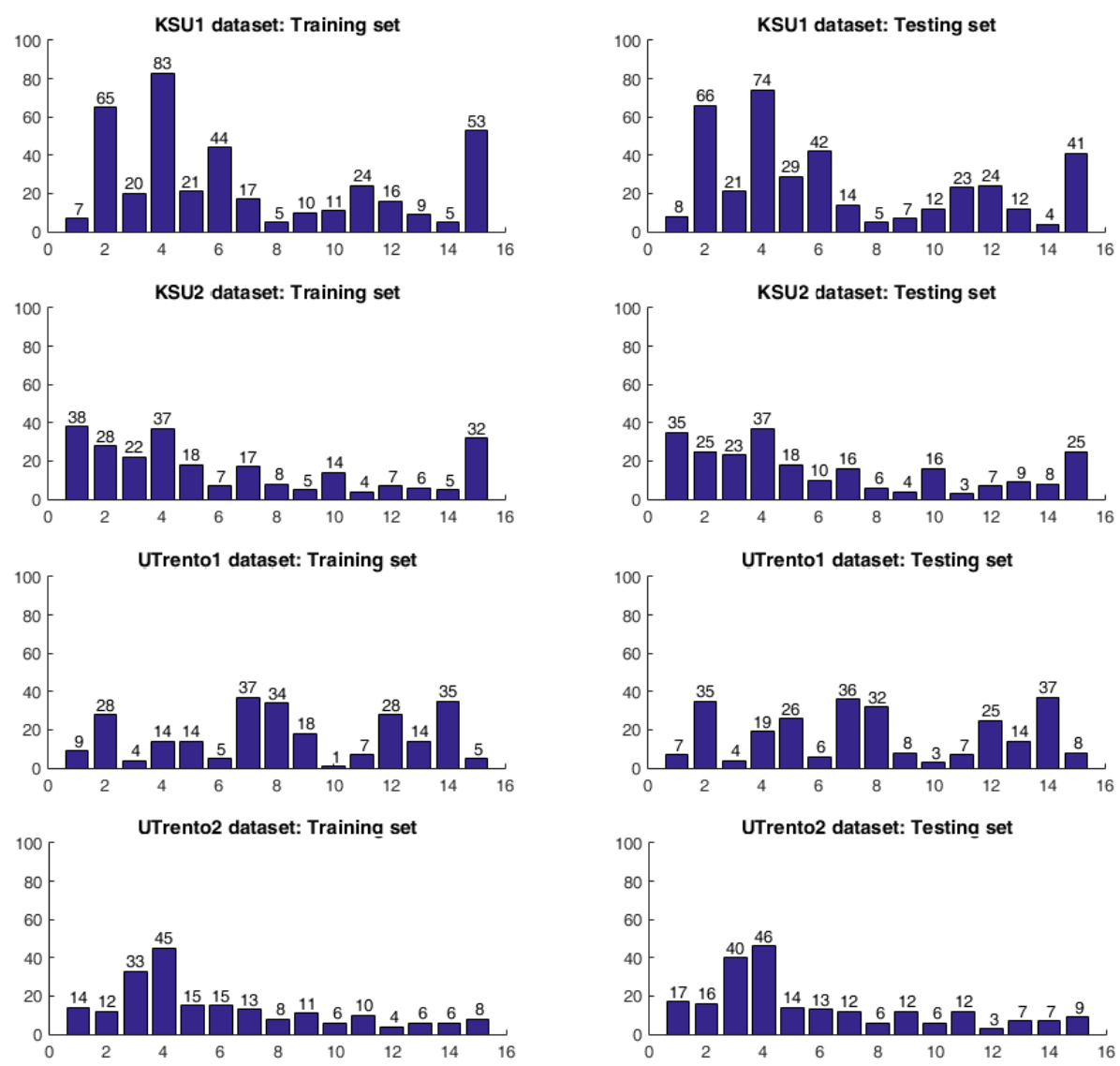

Figure 10. Number of occurrences of objects in each dataset. The $x$ axis represents the object number according to the list given in Table 1. 


\subsection{Experimental Setup}

We implemented the proposed deep CNN solution for image multi-labeling in the Keras environment, which is a high level neural network application programming interface written in Python. We set the number of epochs to 100 and fixed the mini-batch size to 16. Additionally, we set the learning rate of the Adam optimization method to 0.0001 . Regarding the exponential decay rates for the moment estimates and epsilon, we use the following default values: $0.9,0.999$, and 1e-8 respectively.

The datasets contain images of size $640 \times 480$. Rescaling the images to the usual size of $224 \times 224$ is not necessary because the SqueezeNet model is able to accept images of different sizes directly. In fact, this is one the advantages of the SqueezeNet model. Based on the input size of $640 \times 480$, Model 3, presented in Figure 5c, now has a low number of weights between 756,559 to 858,191 only.

The efficiency of the proposed framework is expressed in terms of the measures known as sensitivity (SEN), specificity (SPE), and their average (AVG) defined in $[9,40]$. SEN expresses the probability of correct detection of the presence of an object, while SPE expresses the probability of correct detection of the absence of an object, respectively. They are computed as follows:

$$
\begin{gathered}
\text { SEN }=\frac{\text { True Positives }}{\text { True Positives }+ \text { False Negatives }} \\
\text { SPE }=\frac{\text { True Negatives }}{\text { True Negatives }+ \text { False Positives }} \\
\text { AVG }=\frac{\text { SEN }+ \text { SPE }}{2}
\end{gathered}
$$

All experiments are conducted on an HP-laptop with an Intel Core i7-7700HQ CPU of $2.80 \mathrm{GHz}$, 16 GB of RAM, and the NVIDIA graphics card GeForce GTX 1050 Ti with a 4 GB dedicated memory.

\subsection{Results of the Proposed Deep Solution for Image Multi-Labeling}

First, we present the results for the baseline model (Model 1) shown in Figure 5a based on the SqueezeNet CNN with fixed pre-trained weights. We perform sensitivity analysis for this model by varying the presence threshold $T_{p}$ in a range from 0.1 to 0.8 with a step size of 0.1 and by varying the hidden layer size as the following values $64,128,256$, or 512 . Figure 11 shows the analysis for the different datasets. The first observation in these figures is that a presence threshold value equal to 0.5 does not provide the most accurate results. In fact, a $T_{p}=0.4$ gives us the highest AVG score result overall. Secondly, in terms of the optimal hidden layer size, we can see from the figures that a hidden layer size between 64 and 128 is the most appropriate when considering all datasets.

Next, we present the results of fine-tuning Models 2 and 3 shown in Figure 5b,c, respectively. Figures 12 and 13 gives sensitivity analysis for Models 2 and 3, respectively. As can be seen, Model 3 gives us the best performance in terms of AVG score. Moreover, in general, a presence threshold $T_{p}=0.3$ is the best choice considering all datasets. The number of filters for the CONV2D layer in Model 3 does not significantly affect the results. Therefore, we selected a number equal to 256 that gives good results for most datasets, but keeps processing time per test image low. 


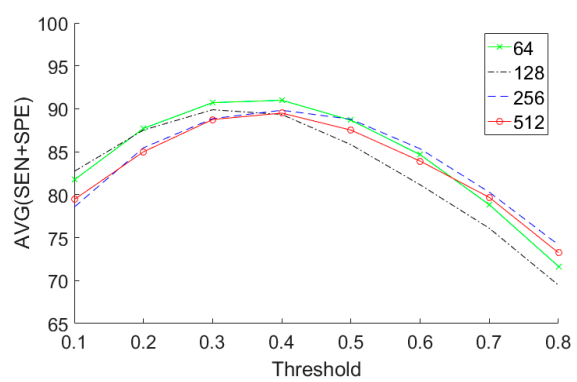

(a)

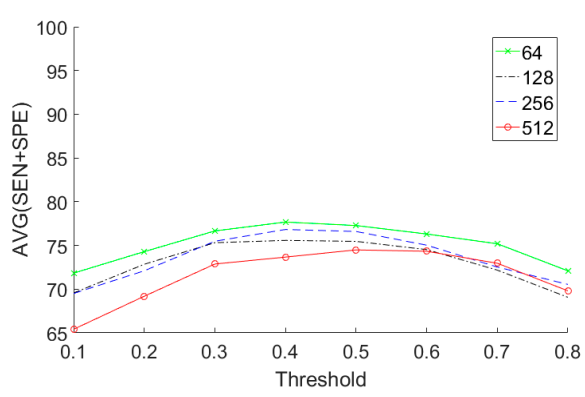

(c)

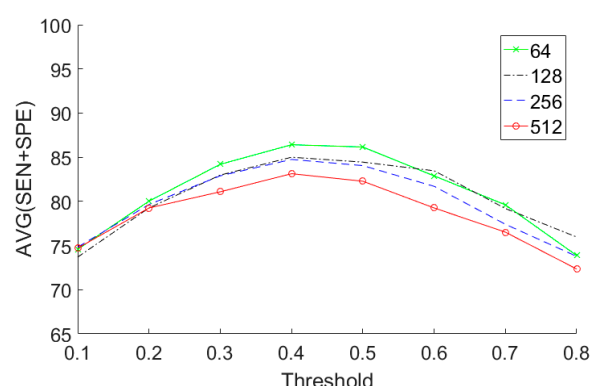

(b)

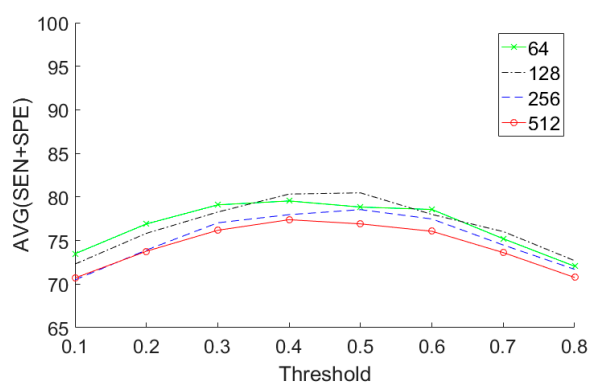

(d)

Figure 11. Effect of the hidden layer size and the presence thresholds $T_{p}$ on the AVG score for the Model 1 (base model): (a) KSU1 dataset, (b) KSU2 dataset, (c) UTrento1, and (d) UTrento2.

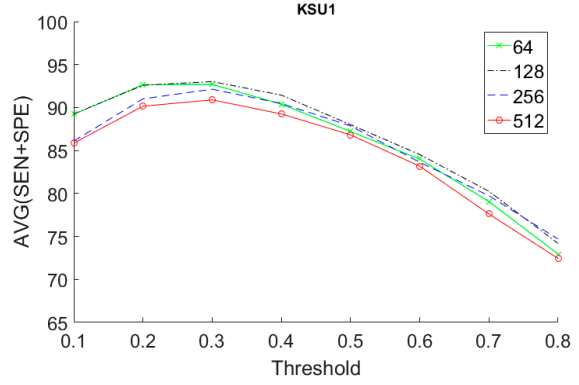

(a)

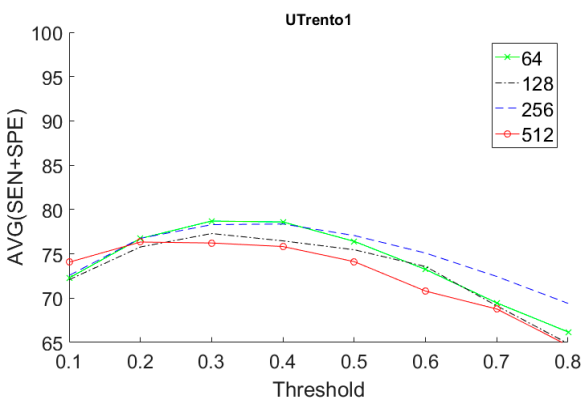

(c)

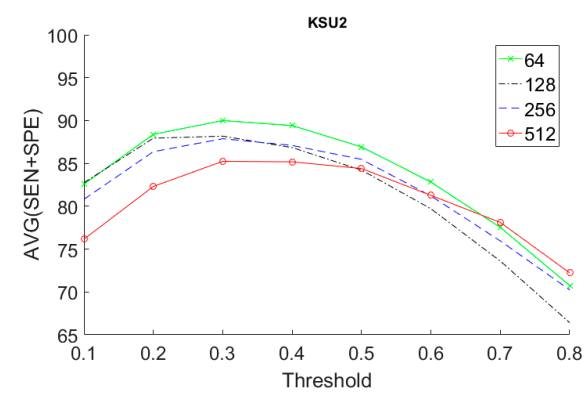

(b)

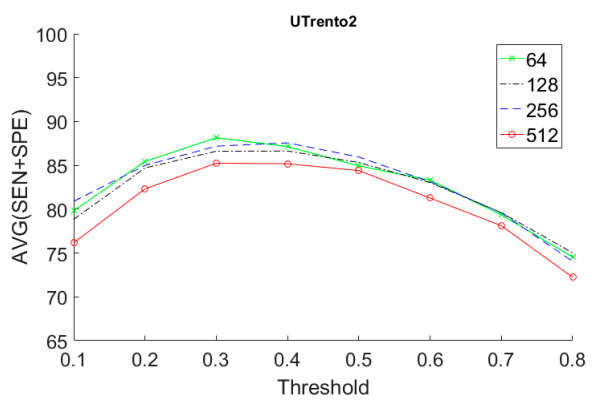

(d)

Figure 12. Effect of the hidden layer size and the presence thresholds $T_{p}$ on the AVG score for Model 2: (a) KSU1 dataset, (b) KSU2 dataset, (c) UTrento1, and (d) UTrento2. 


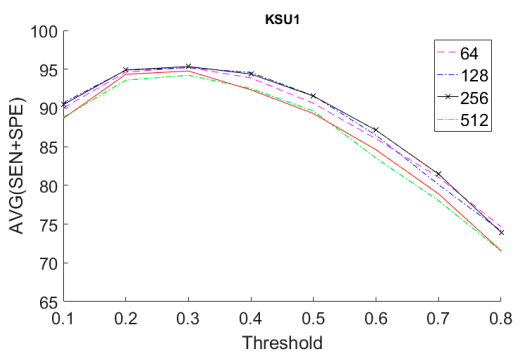

(a)

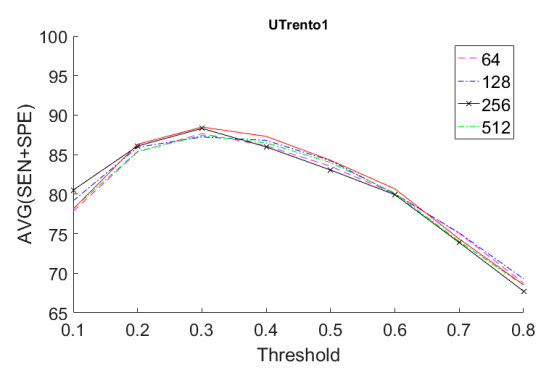

(c)

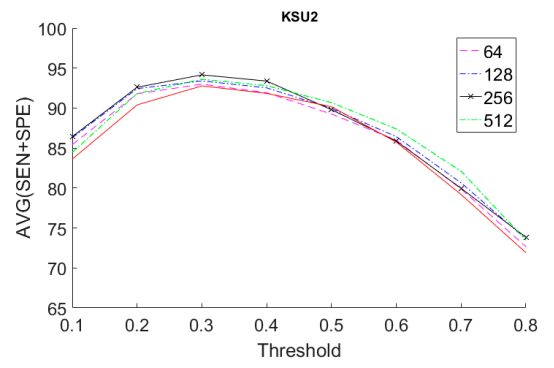

(b)

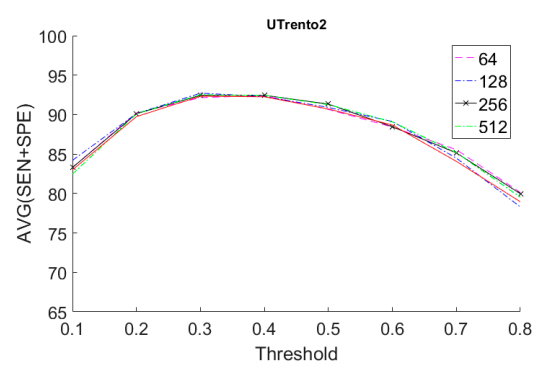

(d)

Figure 13. Effect of the hidden layer size and the presence thresholds $T_{p}$ on the AVG score for Model 3: (a) KSU1 dataset, (b) KSU2 dataset, (c) UTrento1, and (d) UTrento2.

\subsection{Comparison to the State of the Art}

Table 2 presents a comparison between the proposed method and the state-of-the-art methods based on compressive sensing and Gaussian process regression (CS + GP) [9], SURF feature matching, and Gaussian process regression (SURF + GPR) [9], multi-resolution random projection (MR-random-proj) [13], fine-tuning of GoogLeNet [13], and AE fusion of HOG + BOW + LBP features [12]. These results are obtained using the proposed Model 3 with a hidden layer size of 256, and a presence threshold of 0.3 , as suggested by the sensitivity analysis.

Table 2. Comparison to state-of-the-art methods.

\begin{tabular}{clcccc}
\hline \multirow{2}{*}{ Dataset } & \multicolumn{1}{c}{ Method } & SEN & SPE & AVG & Time(s) \\
\hline \multirow{5}{*}{ KSU 1 } & EDCS + GPR [9] & 73.82 & 80.53 & 77.18 & $>1$ \\
\cline { 2 - 6 } & MR random projection [13] & 71.65 & 94.81 & 83.23 & 0.035 \\
\cline { 2 - 6 } & Pre-trained GoogLeNet [13] & 73.56 & 97.95 & 85.75 & 0.064 \\
\cline { 2 - 6 } & Fine-tune improved SqueezeNet [ours] & 94.14 & 96.54 & 95.34 & 0.024 \\
\hline \multirow{5}{*}{ KSU 2 } & EDCS + GPR [9] & 64.46 & 72.91 & 68.69 & $>1$ \\
\cline { 2 - 6 } & MR random projection [13] & 70.00 & 92.33 & 81.16 & 0.037 \\
\cline { 2 - 6 } & Pre-trained GoogLeNet [13] & 67.76 & 95.91 & 81.84 & 0.067 \\
\cline { 2 - 6 } & Fine-tune improved SqueezeNet [ours] & 94.51 & 93.79 & 94.15 & 0.029 \\
\hline \multirow{5}{*}{ UTrento1 } & SURF + GPR [9] & 71.16 & 100.00 & 85.58 & 1.17 \\
\cline { 2 - 6 } & EDCS + GPR [9] & 69.66 & 80.19 & 74.93 & 1.08 \\
\cline { 2 - 6 } & MR random projection [13] & 71.16 & 83.20 & 77.18 & 0.037 \\
\cline { 2 - 6 } & Pre-trained GoogLeNet [13] & 61.79 & 96.55 & 79.17 & 0.068 \\
\cline { 2 - 6 } & AE fusion of HOG + BOW + LBP [12] & 89.51 & 81.3 & 85.41 & 0.022 \\
\cline { 2 - 6 } & Fine-tune improved SqueezeNet [ours] & 88.14 & 88.49 & 88.31 & 0.013 \\
\hline
\end{tabular}


Table 2. Cont.

\begin{tabular}{clcccc}
\hline \multirow{2}{*}{ Dataset } & \multicolumn{1}{c}{ Method } & SEN & SPE & AVG & Time(s) \\
\hline \multirow{5}{*}{ UTrento2 } & SURF + GPR [9] & 77.72 & 100.00 & 88.86 & 1.17 \\
\cline { 2 - 6 } & EDCS + GPR [9] & 70.00 & 90.12 & 80.06 & 1.2 \\
\cline { 2 - 6 } & MR random projection [13] & 77.18 & 91.41 & 84.30 & 0.037 \\
\cline { 2 - 6 } & Pre-trained GoogLeNet [13] & 67.27 & 98.55 & 82.91 & 0.066 \\
\cline { 2 - 6 } & AE fusion of HOG + BOW + LBP [12] & 90.00 & 90.12 & 90.06 & 0.022 \\
\cline { 2 - 6 } & Fine-tune improved SqueezeNet [ours] & 94.91 & 89.97 & 92.44 & 0.014 \\
\hline
\end{tabular}

The results in Table 2 clearly show the superiority of the proposed method for all datasets, not only in terms of AVG accuracy but also in terms of execution time per image. This is mainly due to the effectiveness of the image features extracted with the help of the pre-trained SqueezeNet CNN and the improvements that we have proposed for the architecture.

\section{Discussion}

The results achieved in this study indicate clearly the power of SqueezeNet CNNs in extracting highly descriptive features from images, despite its small size. The improvements that we have proposed for the architecture enhanced the results significantly compared to the typical techniques for utilizing a pre-trained CNN. For example, a pre-trained GoogLeNet has been proposed in [13], but the method of fine-tuning it did not produce good results [13]. The reasons for this are several: 1) GoogLeNet has more weights (more than seven million), which requires larger datasets for training, and 2) GoogLeNet requires that the images be of size $224 \times 224$, which means all images have to be resized from the original size of $640 \times 480$ to $224 \times 244$. This modifies the aspect ratio of the images and causes deterioration in the classification accuracy of the network. When it comes to the SquezeNet architecture used in this study, no resizing of images is needed.

Table 3 presents the AVG scores achieved by Model 3 for all object classes and all datasets where the CONV2D layer size is fixed to 256 and $T_{p}=0.3$. In general, some objects are harder to recognize because of their low occurrence number in the datasets. For example, if we look at object 10 (class of people) in the Utrento1 dataset, we can see that it appears only once in the whole dataset. This explains the recognition rate of $50 \%$ achieved, because with only one example of this object, the network achieves a rate equal to random guessing. In the Utrento2 dataset, the same class of objects (the "people" object) appeared slightly more often ( 8 times) and was recognized $66 \%$ of the time only. Therefore, another reason for the low score for this object class is that it is a complex object with high variability, making it difficult to detect without a large number of training samples.

However, for other objects and despite the low number of occurrence for some of them, their recognition rate is mostly above $80 \%$ (see Figure 14). This is actually due to the fact that most of the objects are man-made and have low intra-class variability in their appearance, especially with the same dataset coming from the same building. This is also explained by the power of the pre-trained $\mathrm{CNN}$ to transfer knowledge from an auxiliary domain with a large dataset to another domain with a smaller dataset.

In the UTrento datasets, there are large barrel geometric distortions of the images. In our opinion, this partly explains the lower AVG scores achieved on these datasets compared to the KSU datasets. For example, in Figure 9, one can observe that the "Board" objects are sometimes located on the edges/corners of the image and hence become distorted, making them harder for the network to recognize. This partly explains the relatively lower AVG score achieved for this object, namely $85.90 \%$ and $79.96 \%$ in the UTrento1 and UTrento2 dataset, respectively, despite their high number of occurrences. 
Table 3. Detailed results per object using Model 3 with the CONV2D layer size $=256$ and $T_{p}=0.3$.

\begin{tabular}{|c|c|c|c|c|c|}
\hline \multicolumn{3}{|c|}{ KSU1 } & \multicolumn{3}{|c|}{ KSU2 } \\
\hline Objects & \# Occ & AVG & Objects & \# Occ & AVG \\
\hline pillar & 7 & 93.75 & board & 38 & 79.10 \\
\hline fire extinguisher/hose & 65 & 92.44 & fire extinguisher/hose & 28 & 92.70 \\
\hline trash can & 20 & 94.41 & trash cans & 22 & 91.73 \\
\hline chairs & 83 & 90.12 & chairs & 37 & 85.22 \\
\hline external door & 21 & 92.89 & external door & 18 & 95.29 \\
\hline hallway & 44 & 93.86 & didactic service machine & 7 & 97.97 \\
\hline self-service & 17 & 94.37 & self-service & 17 & 90.45 \\
\hline reception & 5 & 99.77 & reception & 8 & 98.11 \\
\hline didactic service machine & 10 & 92.2 & cafeteria & 5 & 97.74 \\
\hline display screen & 11 & 99.08 & display screen & 14 & 98.61 \\
\hline board & 24 & 92.92 & pillar & 4 & 99.12 \\
\hline stairs & 16 & 96.7 & stairs & 7 & 97.90 \\
\hline elevator & 9 & 99.59 & elevator & 6 & 98.29 \\
\hline laboratory & 5 & 100.00 & prayer room & 5 & 81.06 \\
\hline internal door & 53 & 84.59 & internal door & 32 & 78.38 \\
\hline- & SEN & 94.14 & - & SEN & 94.51 \\
\hline- & SPE & 96.54 & - & SPE & 93.79 \\
\hline- & AVG & 95.34 & - & AVG & 94.15 \\
\hline \multicolumn{3}{|c|}{ UTrento1 } & \multicolumn{3}{|c|}{ UTrento2 } \\
\hline Objects & \# Occ & AVG & Objects & \# Occ & AVG \\
\hline external window & 9 & 81.49 & stairs & 14 & 98.26 \\
\hline board & 28 & 85.90 & heater & 12 & 94.79 \\
\hline table & 4 & 98.09 & corridor & 33 & 95.54 \\
\hline external door & 14 & 91.03 & board & 45 & 79.96 \\
\hline stair door & 14 & 90.70 & laboratories & 15 & 86.70 \\
\hline access control reader & 5 & 79.17 & bins & 15 & 84.32 \\
\hline office & 37 & 88.33 & office & 13 & 95.80 \\
\hline pillar & 34 & 92.19 & people & 8 & 66.09 \\
\hline display screen & 18 & 90.31 & pillar & 11 & 98.56 \\
\hline people & 1 & 50.00 & elevator & 6 & 99.17 \\
\hline ATM & 7 & 94.33 & reception & 10 & 95.63 \\
\hline chairs & 28 & 84.97 & chairs & 4 & 99.18 \\
\hline bins & 14 & 84.25 & self-service & 6 & 87.86 \\
\hline internal door & 35 & 76.57 & external door & 6 & 95.71 \\
\hline elevator & 5 & 91.25 & display screen & 8 & 95.37 \\
\hline- & SEN & 88.14 & - & SEN & 94.91 \\
\hline- & SPE & 88.49 & - & SPE & 89.97 \\
\hline- & AVG & 88.31 & - & AVG & 92.44 \\
\hline
\end{tabular}

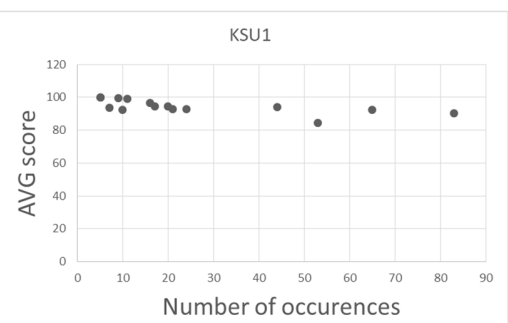

(a)

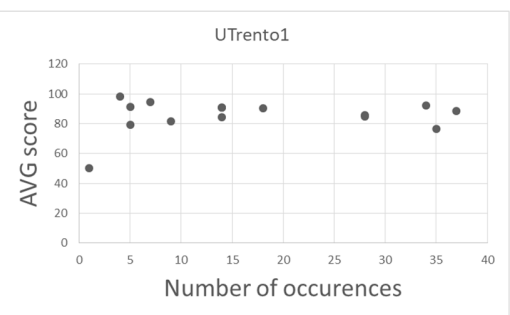

(c)

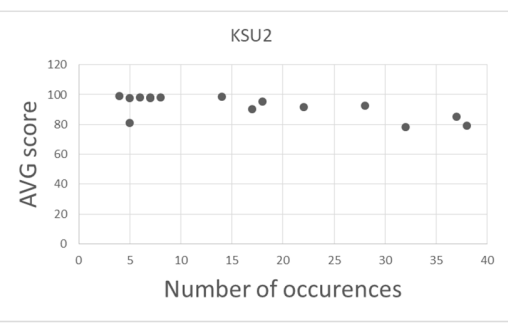

(b)

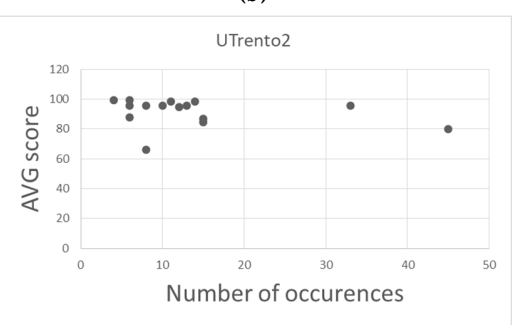

(d)

Figure 14. Distribution of objects with respect to AVG score versus the number of occurrences using Model 3: (a) KSU1 dataset, (b) KSU2 dataset, (c) UTrento1, and (d) UTrento2. 


\section{Conclusions}

In this paper, we have presented an efficient deep learning method for image multi-labeling. This method is a module belonging to a larger system for helping the visually impaired to navigate his environment and recognize objects therein. The system makes the VI person aware of the presence/absence of a given set of objects in his environment. Our solution is based on a light-weight pre-trained CNN called SqueezeNet. We developed an improved version of the SqueezeNet by resetting the weights of the last convolutional layer to new random values, changing the activation function from a ReLU to LeakyReLU, and adding a BatchNormalization layer. Next, using a fine-tuning approach, we successfully trained the proposed deep CNN on four image multi-labeling datasets. Experimental results have shown the effectiveness of our proposed deep CNN, because it has provided significant improvements over the state of the art and has reduced the computational time per image.

Upon inspection of Figure 10, we can observe that the objects have a different number of occurrences in each dataset. This creates a class imbalance problem that negatively affects any classification method. Thus, one future direction of research in order to improve the classification results is to address the class imbalance problem. One way to do this is through augmentation techniques to increase samples in the infrequent classes. Another direction is the use of an ensemble of deep classifiers, where each one is responsible for learning to detect a set of objects that are more balanced in terms of their frequency of occurrence. An intelligent fusion technique could then be used to merge the results of the classifier ensemble.

Author Contributions: Conceptualization, H.A., Y.B., and N.A.; methodology, H.A.; software, B.B.J.; formal analysis, H.A.; investigation, H.A.; writing—original draft preparation, H.A.; writing-review and editing, Y.B. and N.A.; supervision, H.A.; project administration, N.A.; funding acquisition, N.A.

Funding: This work was supported by NSTIP strategic technologies programs, No. 13-MED1343-02, in the Kingdom of Saudi Arabia.

Acknowledgments: This work was supported by NSTIP strategic technologies programs, number (13-MED1343-02) in the Kingdom of Saudi Arabia.

Conflicts of Interest: The authors declare no conflict of interest.

\section{References}

1. World Health Organization. Vision Impairment and Blindness. Available online: https://www.who.int/newsroom/fact-sheets/detail/blindness-and-visual-impairment (accessed on 29 August 2019).

2. Becker, J.V.; Hinton, D.E.; Anderson, H.G., Jr. Braille Computer Monitor. U.S. Patent 6,700,553, 2 March 2004.

3. US Patent Application for Refreshable Braille Display Patent Application (Application \#20130203022 issued 8 August 2013)—Justia Patents Search. Available online: https://patents.justia.com/patent/20130203022 (accessed on 29 August 2019).

4. Thatcher, J. Screen Reader/2-Programmed access to the GUI. In Computers for Handicapped Persons; Springer: Berlin/Heidelberg, Germany, 1994; pp. 76-88.

5. IBM SCREEN READER/2. 23 June 1992. Available online: www-01.ibm.com/common/ssi/cgi-bin/ssialias (accessed on 29 August 2019).

6. Min, B.; Saxena, S.; Steinfeld, A.; Dias, M.B. Incorporating information from trusted sources to enhance urban navigation for blind travelers. In Proceedings of the 2015 IEEE International Conference on Robotics and Automation (ICRA), Seattle, WA, USA, 26-30 May 2015; pp. 4511-4518.

7. Min, B.-C.; Steinfeld, A.; Dias, M.B. How Would You Describe Assistive Robots to People Who Are Blind or Low Vision? In Proceedings of the Tenth Annual ACM/IEEE International Conference on Human-Robot, Portland, OR, USA, 2-5 March 2015; pp. 91-92.

8. Mekhalfi, M.L.; Melgani, F.; Bazi, Y.; Alajlan, N. Toward an assisted indoor scene perception for blind people with image multilabeling strategies. Expert Syst. Appl. 2015, 42, 2907-2918. [CrossRef]

9. Mekhalfi, M.L.; Melgani, F.; Bazi, Y.; Alajlan, N. A compressive sensing approach to describe indoor scenes for blind people. IEEE Trans. Circuits Syst. Video Technol. 2015, 25, 1246-1257. [CrossRef] 
10. Farabet, C.; Couprie, C.; Najman, L.; LeCun, Y. Learning hierarchical features for scene labeling. IEEE Trans. Pattern Anal. Mach. Intell. 2013, 35, 1915-1929. [CrossRef]

11. Brosch, T.; Tam, R. Efficient training of convolutional deep belief networks in the frequency domain for application to high-resolution 2D and 3D images. Neural Comput. 2015, 27, 211-227. [CrossRef]

12. Malek, S.; Melgani, F.; Mekhalfi, M.L.; Bazi, Y. Real-time indoor scene description for the visually impaired using autoencoder fusion strategies with visible cameras. Sensors 2017, 17, 2641. [CrossRef]

13. Mekhalfi, M.L.; Melgani, F.; Bazi, Y.; Alajlan, N. Fast indoor scene description for blind people with multiresolution random projections. J. Vis. Commun. Image Represent. 2017, 44, 95-105. [CrossRef]

14. Alhichri, H.; Jdira, B.B.; Bazi, Y.; Alajlan, N. Multiple Object Scene Description for the Visually Impaired Using Pre-trained Convolutional Neural Networks. In Image Analysis and Recognition; Springer: Berlin/Heidelberg, Germany, 2016; pp. 290-295.

15. Ren, S.; He, K.; Girshick, R.B.; Zhang, X.; Sun, J. Object detection networks on convolutional feature maps. IEEE Trans. Pattern Anal. Mach. Intell. 2015, 39, 1476-1481. [CrossRef]

16. Ren, S.; He, K.; Girshick, R.B.; Sun, J. Faster r-cnn: towards real-time object detection with region proposal networks. IEEE Trans. Pattern Anal. Mach. Intell. 2015, 39, 1137-1149. [CrossRef]

17. Ouyang, W.; Zeng, X.; Wang, X.; Qiu, S.; Luo, P.; Tian, Y.; Wang, K.; Yan, J.; Loy, C.-C.; Tang, X.; et al. DeepID-Net: Object detection with deformable part based convolutional neural networks. IEEE Trans. Pattern Anal. Mach. Intell. 2017, 39, 1320-1334. [CrossRef]

18. Ammour, N.; Alhichri, H.; Bazi, Y.; Benjdira, B.; Alajlan, N.; Zuair, M. Deep learning approach for car detection in UAV imagery. Remote Sens. 2017, 9, 312. [CrossRef]

19. Bengio, Y.; Lamblin, P.; Popovici, D.; Larochelle, H. Greedy layer-wise training of deep networks. In Proceedings of the 19th International Conference on Neural Information Processing Systems; MIT Press: Cambridge, MA, USA, 2006; pp. 153-160.

20. Hinton, G.E.; Osindero, S.; Teh, Y.-W. A fast learning algorithm for deep belief nets. Neural Comput. 2006, 18, 1527-1554. [CrossRef] [PubMed]

21. Lecun, Y.; Bottou, L.; Bengio, Y.; Haffner, P. Gradient-based learning applied to document recognition. Proc. IEEE 1998, 86, 2278-2324. [CrossRef]

22. Mariolis, I.; Peleka, G.; Kargakos, A.; Malassiotis, S. Pose and category recognition of highly deformable objects using deep learning. In Proceedings of the 2015 International Conference on Advanced Robotics (ICAR), Istanbul, Turkey, 27-31 July 2015; pp. 655-662.

23. Krizhevsky, A.; Sutskever, I.; Hinton, G.E. Imagenet classification with deep convolutional neural networks. Commun. ACM 2017, 60, 84-90. [CrossRef]

24. He, K.; Zhang, X.; Ren, S.; Sun, J. Deep residual learning for image recognition. In Proceedings of the 2016 IEEE Conference on Computer Vision and Pattern Recognition (CVPR), Las Vegas, NV, USA, 27-30 June 2016; pp. 770-778.

25. Alhichri, H.; Othman, E.; Zuair, M.; Ammour, N.; Bazi, Y. Tile-Based Semisupervised Classification of Large-Scale VHR Remote Sensing Images. J. Sens. 2018, 2018, 14. [CrossRef]

26. Shelhamer, E.; Long, J.; Darrell, T. Fully convolutional networks for semantic segmentation. In Proceedings of the 2015 IEEE Conference on Computer Vision and Pattern Recognition (CVPR), Boston, MA, USA, 7-12 June 2015; pp. 3431-3440.

27. Goodfellow, I.; Bengio, Y.; Courville, A. Deep Learning; The MIT Press: Cambridge, MA, USA, 2016.

28. Krizhevsky, A.; Sutskever, I.; Hinton, G.E. ImageNet Classification with Deep Convolutional Neural Networks. In Advances in Neural Information Processing Systems 25; Pereira, F., Burges, C.J.C., Bottou, L., Weinberger, K.Q., Eds.; Curran Associates, Inc.: Red Hook, NY, USA, 2012; pp. 1097-1105.

29. Simonyan, K.; Zisserman, A. Very deep convolutional networks for large-scale image recognition. arXiv 2014, arXiv:14091556.

30. Szegedy, C.; Liu, W.; Jia, Y.; Sermanet, P.; Reed, S.; Anguelov, D.; Rabinovich, A.; Erhan, D.; Vanhoucke, V.; Google Inc; et al. Going deeper with convolutions. In Proceedings of the 2015 IEEE Conference on Computer Vision and Pattern Recognition (CVPR), Boston, MA, USA, 7-12 June 2015; pp. 1-9.

31. Chatfield, K.; Simonyan, K.; Vedaldi, A.; Zisserman, A. Return of the devil in the details: Delving deep into convolutional nets. arXiv 2014, arXiv:14053531.

32. Azizpour, H.; Razavian, A.S.; Sullivan, J.; Maki, A.; Carlsson, S. Factors of transferability for a generic convnet representation. IEEE Trans. Pattern Anal. Mach. Intell. 2014, 38, 1790-1802. [CrossRef] 
33. Nogueira, R.F.; de Lotufo, R.A.; Machado, R.C. Fingerprint liveness detection using convolutional neural networks. IEEE Trans. Inf. Forensics Secur. 2016, 11, 1206-1213. [CrossRef]

34. Gao, C.; Li, P.; Zhang, Y.; Liu, J.; Wang, L. People counting based on head detection combining Adaboost and CNN in crowded surveillance environment. Neurocomputing 2016, 28, 108-116. [CrossRef]

35. Bengio, Y.; Courville, A.; Vincent, P. Representation learning: A review and new perspectives. IEEE Trans. Pattern Anal. Mach. Intell. 2013, 35, 1798-1828. [CrossRef]

36. Schmidhuber, J. Deep learning in neural networks: An overview. Neural Netw. 2015, 61, 85-117. [CrossRef] [PubMed]

37. Ioffe, S.; Szegedy, C. Batch normalization: Accelerating deep network training by reducing internal covariate shift. In Proceedings of the 32nd JMLR International Conference on Machine Learning, Lille, France, 6-11 July 2015; pp. 448-456.

38. IDS Imaging Development Systems GmbH. Industrial Cameras_IDS Imaging Development Systems GmbH. Available online: https://en.ids-imaging.com/ (accessed on 21 October 2019).

39. RMA Electronics, Inc. Kowa LM4NCL 1/2" 3.5mm F1.4 Manual Iris C-Mount Lens; RMA Electronics, Inc.: Hingham, MA, USA; Available online: https://www.rmaelectronics.com/kowa-lm4ncl/ (accessed on 21 October 2019).

40. Razavi, N.; Gall, J.; Van Gool, L. Scalable multi-class object detection. In Proceedings of the 2011 IEEE Conference on Computer Vision and Pattern Recognition, Providence, RI, USA, 20-25 June 2011; pp. 1505-1512.

(C) 2019 by the authors. Licensee MDPI, Basel, Switzerland. This article is an open access article distributed under the terms and conditions of the Creative Commons Attribution (CC BY) license (http://creativecommons.org/licenses/by/4.0/). 